\title{
Congenital Cataract Surgery: Our Four Years' Experience in Prince Hamza Hospital (Amman-Jordan)
}

\author{
Raed Shatnawi ${ }^{1,2}$, Mohammad Abu-Ain ${ }^{1,2}$, Motasem Al-Latayfeh ${ }^{1,2} \&$ Basel Baarah $^{3}$ \\ ${ }^{1}$ School of Medicine, Hashemite University, Zarka, Jordan \\ ${ }^{2}$ Department of Ophthalmology, Prince Hamza Hospital, Amman, Jordan \\ ${ }^{3}$ Department of Ophthalmology, King Hussein Medical Center, Royal Medical Services, Amman, Jordan \\ Correspondence: Raed Shatnawi, MD, FRCS (Glasg), Assisitant Professor of Ophthalmology, P.O.Box 1691, \\ Tarek, Amman 11947, Jordan. Tel: 962-79-082-4369. E-mail: raed972@yahoo.com
}

Received: December 3, 2018 Accepted: February 27, 2019 Online Published: March 15, 2019

doi:10.5539/gjhs.v11n4p85 URL: https://doi.org/10.5539/gjhs.v11n4p85

\begin{abstract}
Purpose: The study has examined the problems encountered during management of cataract surgery during clinical practice within a developing country.

Methods: A retrospective study was conducted by recruiting patients with the diagnosis of congenital cataract operated between 2011 and 2014. Intraocular lens was implanted, when the corneal diameter was $10 \mathrm{~mm}$ or more, which approximately corresponded to the capsular bag diameter, regardless of the patients' age.

Results: The results showed that 13 were aphakic and none of them developed visual axis opacification. Eleven out of the 49 pseudophakic patients needed 2 or more surgeries to clear visual axis opacification; whereas, 25 out of 49 pseudophakic patients received hydrophobic intraocular lens. However, 3 of them (12\%) developed visual axis opacification. The remaining 24 patients received hydrophilic intraocular lens, where 8 of them (33\%) developed visual axis opacification. There was increased incidence of visual axis opacification as a result of pseudophakia that required a second surgery, which delayed their visual rehabilitation.
\end{abstract}

Conclusion: Hydrophobic intraocular lenses are better used because of their lower risk to induce visual axis opacification.

Keywords: congenital cataract, implantation, surgery

\section{Introduction}

The occurrence of congenital cataract has been considered as among leading causes of childhood blindness in the developing countries (H. Lin et al., 2014). Cataract is associated with clouding of eye's natural lens as age of an individual progresses. The congenital cataract commonly occurs among newborn infants as a result of infection, trauma, diabetes, drug reactions, inherited tendencies, inflammation, and metabolic problems (Lambert, 2004). Congenital cataract may even develop as a reaction against tetracycline antibiotic, taken by pregnant women to treat infection (Ventura, Sampaio, Ventura, Ventura, \& Nosé, 2013). The abnormalities associated with congenital cataracts occur by the formation of essential proteins that maintain the transparency of eye's natural lens.

In recent years, the enhancements in surgical techniques have a great impact on the outcomes, following surgery for cataracts among the infants. However, there is inevitable rise in the complications, which can originate intra-operatively, shortly, or in the long-term follow-up period post-operatively. The most common complication associated with this surgery is secondary opacification of the visual axis that is particularly linked with the intraocular lens (IOL) implantation among infants due to cortex re-proliferation (Plager et al., 2014). IOL means more inflammation, secondary membranes, IOL decentration, and more surgeries. Awareness about the potential disorders that are unusual to the infant eyes can assist surgeons to prevent the adverse outcomes in several cases. It may help the surgeons to identify and treat effectively (Plager, 2017).

Congenital cataracts have remained one of the major causes over the past decades of treatable childhood blindness (Lim, Rubab, Chan, \& Levin, 2010). The aetiology of congenital cataract is multifactorial; however, the most important risk factor is the age of children. The major factors governing the ultimate visual outcomes, concerning congenital cataract surgery include; management of the posterior capsule, aggressive amblyopia therapy, and 
refractive management. The rate of posterior capsular opacification has been reduced by using many surgical techniques. It has been observed that posterior chamber IOL implantation with anterior vitrectomy and posterior capsulotomy is the widely accepted surgical process for managing congenital cataracts (Lim et al., 2010). The age is controversial at which the posterior capsulotomy and anterior vitrectomy should be performed (Latif, Shakir, Zafar, Rizvi, \& Naz, 2014). It has been observed that 1-15 per thousand children have suffered from this pathology, and there is 10 -fold increase in its prevalence rate in the developing regions as compared to the developed regions (D. Lin et al., 2015). Paediatric cataract surgery is still the first line of treatment, despite of its poor visual results (Lenhart et al., 2015). Moreover, paediatric cataract surgery is associated with serious intraoperative and postoperative consequences with several comorbidities. The intraoperative and postoperative consequences include; posterior capsular opacity, strabismus, glaucoma, and nystagmus. Moreover, the proper and timely treatment is restricted to the developed regions, due to lack of equipment, techniques, lack of funding, and strategic policies from the Health Ministry.

The surgical management of congenital cataract is a challenging task that needs effective instrumentation and lengthy follow ups. There is much controversy in the optimal time and approach needed to be used during congenital cataract surgery. Moreover, the population based information on surgical treatments and epidemiology are critical for enhancing the treatment and prevention programs, particularly in developing regions. The Prince Hamza Hospital in Amman-Jordan is the largest and only referral hospital for the Ministry of Health in Jordan that provides pediatric ophthalmology service in the country. However, published reports based on the incidence and prevalence of congenital cataracts in Jordan are unavailable. The eye of an infant behaves inversely to an eye of adult; and this is the reason that congenital cataract surgery must be performed only in the centers, which are appropriately equipped to perform the demanding process. The aim of congenital cataract surgery is to be performed at an age, which results in optimized visual outcome and minimizes the risk of glaucoma development in later age. Delay in cataract surgery may result in worse visual outcomes. There are less chances of vision to be regained, if the cataract is not removed during the first few months of life among the infants suffering congenital cataract. Therefore, the main aim of the study was to assess the congenital cataract surgery in Prince Hamza Hospital (Amman-Jordan), which is a developing region to deal with the complications of cataract surgery. Moreover, the study has mainly focused on the different factors that modulate the anatomical and functional results after congenital cataract after its surgery.

\section{Material and Methods}

\subsection{Study Design and Sampling}

A retrospective study has been conducted by recruiting 62 patients, who were diagnosed with congenital cataract and operated by surgeon between years 2011 and 2014 in Prince Hamza Hospital. All these patients were operated by a single surgeon. Intraocular lens (IOL) was implanted when the corneal diameter was $10 \mathrm{~mm}$ or more, which almost corresponded to the capsular bag diameter. IOLs were used because it capable of reducing the dependence on the compliance in contrast with other optical devices, including contact lenses and aphakic glasses by providing the partial correction at least.

\subsection{Inclusion and Exclusion Criteria}

The patients with gestational age $>37$ weeks and diagnosed with dense opacity covering the entire lens were recruited in the study. However, the patients with ocular trauma, persistent hyperplastic primary vitreous, history of glaucoma, corneal disorder, Lower syndrome, and rubella were excluded. The types of cataracts included in the study were congenital, present at birth or possibly post traumatic. Moreover, the patients, who were not able to complete the follow up and did not have normal dilation of pupil were not included in the study. It was necessary for all the recruited patients to sign an informed written consent before getting involved in the study procedure.

\subsection{Data Collection and Analysis}

The study has described the local experience at Prince Hamza Hospital, which is the only congenital cataract service in the Ministry of Health of Jordan. The sample of the study was comprised of infants, less than 1 year up to 8 years, having surgery through limbal approach as follows;

Primary posterior capsulotomy (PPC) + anterior vitrectomy (AV) + IOL (in the bag).

Intracameral injection of dexamethasone and 4 quadrants subconjunctival injection of gentamicin/dexamethasone were given at the conclusion of surgery to reduce post-operative inflammation. The data regarding age of patient at time of surgery, type of surgery, implantation of intraocular lens (IOL), number of surgeries, and complications have been collected for each patient. Hydrophilic or hydrophobic acrylic IOLs were used as two types of bio-compatibility materials because of its long history of clinical practice and safe to be used for intraocular 
implantation. The study has identified the type of IOL (hydrophobic acrylic IOL or hydrophilic acrylic IOL) performed on the patient and compared the outcomes of both procedures.

\section{Results}

Among 62 patients suffering from congenital or developmental cataracts, 32 patients were males and 30 patients were females. Seventeen patients were one year old or younger at the time of surgery; of whom 7 were left aphakic and 10 pseudophakic. Of the aphakic group, no one developed secondary membranes or unusual inflammatory response. In order to clear the visual axis, two of the pseudophakic patients needed a second surgery. Forty nine patients had pseudophakia; of whom, 25 patients received hydrophobic IOL and 24 patients received hydrophilic IOL. Concerning the hydrophobic group, 3 patients (12\%) developed visual axis opacification (VAO) that clarified the requirement of second surgery. Of the hydrophilic group, 8 patients (33\%) have developed VAO, which needed second surgery. one patient of the hydrophilic group needed 3 surgeries, ending in an explant of the IOL to control the inflammation.

Of the total 62 patients, 13 were aphakic and no one developed VAO. Moreover, 11 out of 49 pseudophakic patients needed 2 or more surgeries to clear the VAO. Twenty five out of the 49 pseudophakic patients received hydrophobic IOL; while, 3 of them (12\%) developed VAO. The remaining 24 patients received hydrophilic IOL and 8 of them (33\%) developed VAO clarifying the need of second surgery. 22 patients were lost from follow ups during the review period, but they were also analyzed, which showed that 8 of them were aphakic and 14 were pseudophakic.

Table 1. Demography

\begin{tabular}{ll}
\hline Males & $32(52 \%)$ \\
Females & $30(48 \%)$ \\
Bilateral & $41(66 \%)$ \\
Unilateral & $21(34 \%)$ \\
Less than 1 year & $17(27 \%)$ \\
Aphakic & $13(21 \%)$ \\
Pseudophakic & $49(79 \%)$ \\
Hydrophobic IOL & $25(51 \%)$ \\
Hydrophilic IOL & $24(49 \%)$ \\
\hline
\end{tabular}

Table 1 has shown that a total of 17 patients $(27 \%)$ were less than 1 year; however, 7 patients were aphakic. Two of them needed surgery for glaucoma ( 1 patient was microphthalmic and the other one presented with high IOP before the cataract surgery). None of the patients developed secondary membranes or unusual inflammatory response. Ten patients were pseudophakic; of whom, 6 received hydrophobic IOL and 4 received hydrophilic IOL. In aphakic group, no patient has developed VAO. In pseudophakic group; however, 2 patients out of 10 have developed VAO. It has been observed that VAO risk with hydrophobic IOL is less.

\subsection{Aphakia vs. Pseudophakia}

The results have shown that 13 patients were aphakic that is $0 \%$ developed visual axis opacification. Aphakic glasses and aphakic contact lenses were used for the optical correction of aphakia. They received visual rehabilitation shortly after the surgery. Forty nine patients were pseudophakic, of whom $22 \%$ have developed significant VAO requiring 2 or more surgeries to clear the visual axis, resulting in delay of visual rehabilitation.

\subsection{Complications Encountered}

Complications encountered were usually related to VAO, along with other complications including IOL decentration (4 cases), dropped IOL ( 1 case), endophthalmitis ( 1 case), and retinal detachment (1 case: this patient was lost from F/U for 3 years with a severe deep inflammatory reaction). Glaucoma was not observed as a major complication in the study. Some patients with bilateral cataracts was lost from $\mathrm{F} / \mathrm{U}$ after doing one eye to reappear again after 2 or 3 years.

\section{Discussion}

There is significant advancement in the improvement of surgical techniques after congenital cataract surgery over 
recent decades. Prince Hamza Hospital in Amman-Jordan is one of the largest referral hospitals and the only pediatric ophthalmology service for the whole Ministry of Health hospitals in Jordan. Pseudophakia resulted in higher incidence of VAO, required a second surgery, and delayed their visual rehabilitation. Pseudophakia resulted in higher number of patients being lost from follow up, which may be a reason to perform bilateral surgery by taking the necessary measures to prevent postoperative infection. The present study has shown that complications encountered after cataract surgeries relate to VAO, IOL decentration (4 cases), dropped IOL (1 case), endophthalmitis (1 case), and retinal detachment. On the contrary, a study revealed that apart from good visual outcomes, an acceptable rate of various post-operative complications is observed in micro-ophthalmic eyes as a sign of early surgical intervention (Praveen, Vasavada, Shah, Khamar, \& Trivedi, 2015). Similarly, a study conducted in China by D. Lin et al. (2015) reported a greater number of congenital cataracts among patients and an augmented incidence of congenital cataracts after the establishment of Childhood Cataract Program of the Chinese Ministry of Health $(\mathrm{CCPMOH})$. The ten-year overview presented the increasing trends in the hospital-based occurrence of congenital cataracts along with more reduction of the age at operation, which possibly highlighted the influence of CCPMOH and offer detailed helpful information (D. Lin et al., 2015). The results were consistent with the present study as it shows association with congenital cataracts and a prominent foundation of the treatment and prevention of this disease of childhood blindness.

Newer lens technology, advances in microsurgical techniques, and an enhanced knowledge of the refractive eye growth has paved the manner for the implantation of IOL in patients. A long term clear axis was provided by the surgical technique by avoiding the development of secondary membrane or posterior capsular opacification. Anesthesia was given with suitable equipment. A vitrectomy instrument was used to deal with posterior capsule. The recent clinical and laboratory research has highlighted the need of modifying treatment protocols to implement satisfactory binocular visual experience during infancy period. Supplementary factors that could influence stereo-acuity outcomes comprise of operation time, post-surgical consequences, treatment modality, and techniques for measuring stereo-acuity. Similar to the present study, Magli et al. (2016) conducted a retrospective interventional research to evaluate long-term functional consequences after congenital and developmental cataracts surgery. The results showed improved distance and near BCVA after the surgery for developmental cataracts. However, the occurrence of strabismus and myopic shift was observed at lower rates. The analysis showed better functional results by postsurgical data in developmental cataracts as compared to congenital cataracts. Various conditions like prenatal infection, prenatal drug exposure, prenatal ionizing radiation, and prenatal maternal diabetes have been linked with cataract (Yorston, 2004).

The present study has depicted that glaucoma was not a major complication; whereas, one of the patients with bilateral cataracts were lost from $\mathrm{F} / \mathrm{U}$ after doing one eye to reappear again after 2 or 3 years. A similar study conducted by Birch and O'Connor (2017) suggested that visual deprivation because of congenital cataracts dislocate binocular cortical development during acute period, normally resulting in reduced stereo-acuity. Another study conducted by Cvekl, Friedman, and Semina (2014) explained the formation of lens through DNA-binding transcription factors; specifically represented in the lens, current strategies and assistances to the human paediatric cataracts in clinical studies and human genetic studies as well. The results have helped in the identification of original cataract-associated genes to have a clear understanding of molecular basis related to these defects and treatments. Surgical management of congenital cataract is a challenging task as it needs sophisticated instrumentation and lengthy follow up (Loganathan, Stephen, Sivaranjani, \& Rao, 2016). A study by Ram, Jain, Agarwal, and Kumar (2014) suggested that comparable complications might be probable among infants with hydrophobic acrylic lenses and polymethyl methacrylate (PMMA). Further surgical intervention for posterior capsular opacification (PCO) is needed by the children implanted with PMMA IOLs. On the contrary, the results of present study stated that hydrophobic IOLs result in a lower incidence of VAO. However, best-corrected visual acuity improvement was obtained by Ventura et al. (2013) that resulted in congenital cataract surgery in microphthalmic eyes from primary intraocular lens implantation. Moreover, the reported cases showed no intraoperative complications and minimal postoperative complications. Primary implantation of IOL in the first year of life can be performed by taking necessary precautions. These results are consistent with the findings deduced in the present study.

A study by To et al. (2014) observed that cataract surgery was capable of enhancing vision-related quality of life (VRQOL) in bilateral cataract patients. It was observed that VRQOL is affected by contrast sensitivity, stereopsis, and visual acuity after undergoing cataract surgery. Similar to the present study, Plager et al. (2014) suggested that aphakic patients in the Infant Aphakia treatment study were instructed to avoid having a secondary IOL implanted during 5 years. However, IOL exchange was observed in 3 patients prior to 5 years of age, who developed myopic refractive errors due to the amplification of the axial length of the eye. There was no significant difference in the 
visual prognosis between the aphakic and pseudophakic groups. Moreover, the results have clearly shown that pseudophakia resulted in higher incidence of significant VAO.

\section{Conclusion}

The present study has investigated different factors that modulating the anatomical and functional results after congenital cataract after its surgery. Bilateral surgery must be promoted in the developing countries, as there is an urgent need for the amenities to track patients, who have not come for further follow-ups. Pseudophakia resulted in higher number of patients, being lost from follow-up. However, the study suffers from significant limitations because being a retrospective study the data collected is neither complete nor accurate. The study has not mentioned the visual outcomes of cataract surgery because of lack of accurate documentation. Despite of these limitations, the study has successfully highlighted an important health issue among Jordanian population with significant impact of different socioeconomic factors on the final surgical outcome. The study reported a prior need for a service to track patients, who are lost from follow-up. Bilateral simultaneous surgery should be encouraged, taking proper precautions.

\section{Ethical Approval}

All procedures performed in studies involving human participants were in accordance with the ethical standards of the institutional and/or national research committee.

\section{Informed Consent}

Informed consent was obtained from all individual participants included in the study.

\section{Acknowledgement}

Raed Shatnawi is very thankful to all the associated personnel in any reference that contributed in/for the purpose of this research.

\section{Funding}

This study is not associated with any funding.

\section{Conflict of Interest}

The authors declare that there are no competing or potential conflicts of interest.

\section{References}

Birch, E. E., \& O'Connor, A. R. (2017). Stereopsis Outcomes in Children Treated for Congenital Cataracts Congenital Cataract (pp. 225-235). Springer.

Cvekl, A., Friedman, I. B., \& Semina, E. V. (2014). Lens-Specific Transcription Factors and Their Roles in Diagnosis and Treatment of Human Congenital Cataract Lens Epithelium and Posterior Capsular Opacification (pp. 105-130). Springer.

Lambert, S. (2004). Treatment of congenital cataract. BMJ Publishing Group Ltd.

Latif, K., Shakir, M., Zafar, S., Rizvi, S. F., \& Naz, S. (2014). Outcomes of congenital cataract surgery in a tertiary care hospital. Pakistan Journal of Ophthalmology, 30(1).

Lenhart, P. D., Courtright, P., Wilson, M. E., Lewallen, S., Taylor, D. S., Ventura, M. C., . . Kruger, S. (2015). Global challenges in the management of congenital cataract: proceedings of the 4th International Congenital Cataract Symposium held on March 7, 2014, New York, New York. Journal of American Association for Pediatric Ophthalmology and Strabismus, 19(2), e1-e8. https://doi.org/10.1016/j.jaapos.2015.01.013

Lim, Z., Rubab, S., Chan, Y. H., \& Levin, A. V. (2010). Pediatric cataract: the Toronto experience-etiology. American journal of ophthalmology, 149(6), 887-892. https://doi.org/10.1016/j.ajo.2010.01.012

Lin, D., Chen, J., Lin, Z., Li, X., Wu, X., Long, E., . . Chen, W. (2015). 10-year overview of the hospital-based prevalence and treatment of congenital cataracts: the CCPMOH experience. PloS one, 10(11), e0142298. https://doi.org/10.1371/journal.pone.0142298

Lin, H., Yang, Y., Chen, J., Zhong, X., Liu, Z., Lin, Z., . . Zhang, X. (2014). Congenital cataract: prevalence and surgery age at Zhongshan Ophthalmic Center (ZOC). PloS one, 9(7), e101781. https://doi.org/10.1371/journal.pone.0101781

Loganathan, M., Stephen, J. R., Sivaranjani, S., \& Rao, V. A. (2016). Prevalence And Causes For Blindness Among Rural Population In Coastal Villages Of Puducherry And Tamilnadu. Trauma, 132, 1.18. 
Magli, A., Forte, R., Carelli, R., Magli, G., Esposito, F., \& Torre, A. (2016). Long-term follow-up after surgery for congenital and developmental cataracts. Paper presented at the Seminars in ophthalmology.

Plager, D. A. (2017). Complications Following Congenital Cataract Surgery Congenital Cataract (pp. 173-179). Springer.

Plager, D. A., Lynn, M. J., Buckley, E. G., Wilson, M. E., Lambert, S. R., DuBois, L., . . Everett, D. F. (2014). Complications in the first 5 years following cataract surgery in infants with and without intraocular lens implantation in the Infant Aphakia Treatment Study. American journal of ophthalmology, 158(5), 892-898. e892. https://doi.org/10.1016/j.ajo.2014.07.031

Praveen, M. R., Vasavada, A. R., Shah, S. K., Khamar, M. B., \& Trivedi, R. H. (2015). Long-term postoperative outcomes after bilateral congenital cataract surgery in eyes with microphthalmos. Journal of Cataract \& Refractive Surgery, 41(9), 1910-1918. https://doi.org/10.1016/j.jcrs.2015.10.005

Ram, J., Jain, V. K., Agarwal, A., \& Kumar, J. (2014). Hydrophobic acrylic versus polymethyl methacrylate intraocular lens implantation following cataract surgery in the first year of life. Graefe's Archive for Clinical and Experimental Ophthalmology, 252(9), 1443-1449. https://doi.org/10.1007/s00417-014-2689-0

To, K. G., Meuleners, L. B., Fraser, M. L., Van Do, D., Van Duong, D., Huynh, V.-A. N., . . Do Nguyen, N. (2014). The impact of cataract surgery on vision-related quality of life for bilateral cataract patients in Ho Chi Minh City, Vietnam: a prospective study. Health and quality of life outcomes, 12(1), 16. https://doi.org/10.1186/1477-7525-12-16

Ventura, M. C., Sampaio, V. V., Ventura, B. V., Ventura, L. O., \& Nosé, W. (2013). Congenital cataract surgery with intraocular lens implantation in microphthalmic eyes: visual outcomes and complications. Arquivos brasileiros de oftalmologia, 76(4), 240-243. https://doi.org/10.1590/S0004-27492013000400011

Yorston, D. (2004). Surgery for congenital cataract. Community eye health, 17(50), 23.

\section{Appendix A}

\begin{tabular}{|c|c|c|c|c|c|}
\hline SEX & AGE NOW & LENS & N OF SX & UNI/BIL & $\begin{array}{l}\text { NOTES } \\
\text { COMPLICATIONS } \\
\text { +1ST REFRACTION AFTER SX } \\
\text { +LAST REFRACTION }\end{array}$ \\
\hline Male & & Aphakia & 1 & BIL & decided to take him for lost follow up \\
\hline Male & 3 & Aphakia & 1 & BILATERAL & \\
\hline Female & 5 & Aphakia & 1 & BIL & $\begin{array}{l}\text { L:total RD, with IOL } \\
\text { R:aphakia, micor-opthalmia }\end{array}$ \\
\hline Female & 6 & Aphakia PHPV & 1 & BIL & $\begin{array}{l}\text { phpv } \\
\text { left phthetic } \\
\text { r cloboma } \\
\text { no sx for RE }\end{array}$ \\
\hline Male & 4 & Aphakia PHPV & 1 & BIL & PHPV \\
\hline Female & 3 & Philic & 1 & UNI & \\
\hline Female & 6 & Philic & 2 & UNI & \\
\hline Female & 11 & Philic & 1 & BIL & \\
\hline Female & 11 & Philic & 2 & BIL & mental retardation \\
\hline Female & 8 & Phobic & & & \\
\hline Female & 3 & Aphakia & 2 & $\mathrm{BIL}$ & bilateral micro-opthalmia developed glucoma \\
\hline Male & 12 & Philic & 1 & BIL & mental retardation \\
\hline Male & 14 & Philic & 1 & & marfan syn no IOL \\
\hline
\end{tabular}




\begin{tabular}{|c|c|c|c|c|c|}
\hline Female & 2 & Philic & 1 & BIL & down syndrome \\
\hline Female & 4 & Phobic & 1 & BIL & \\
\hline Female & & Phobic & & BIL & decided to take him for lost follow up \\
\hline Female & 6 & Phobic & 1 & BIL & $\begin{array}{l}\text { did RE lost follow up } 2 \text { y } \\
\text { did another sx to same eye } \\
\text { did LE } 2 \text { y }\end{array}$ \\
\hline Female & 7 & Aphakia & 1 & UNIL & micro-ophthalmia \\
\hline Male & $16 \mathrm{M}$ & Aphakic & 2 & BIL & $\begin{array}{l}\text { DEVELOPED GLUCOMA } \\
\text { AFTER SX } \\
\text { NEEDED AHMED VALVE }\end{array}$ \\
\hline Male & 4 & Philic & & BIL & did one eye \\
\hline Female & 6 & Philic & 1 & UNIL & \\
\hline Male & 8 & Philic & 1 & BIL & \\
\hline Female & $13 \mathrm{~m}$ & Aphakia & 1 & BIL & \\
\hline Female & $16 \mathrm{~m}$ & Aphakia & 1 & UNI & \\
\hline Male & 5 & Aphakia & 1 & $\mathrm{BI}$ & $\begin{array}{l}\text { mild esoptopia } \\
\text { no nystagmus } \\
\text { last } R E F+11.0 \text { on }\end{array}$ \\
\hline Female & 8 & Aphakia & 1 & UNI & truamatic catract \\
\hline Male & $15 \mathrm{M}$ & Aphakia & 1 & UNI & \\
\hline Female & $20 \mathrm{M}$ & Philic & 2 & BIL & \\
\hline Female & 3 & Philic & 1 & UNI & \\
\hline Male & 7 & Philic & 2 & BIL & \\
\hline Male & 5 & Philic & 1 & BILATERAL & \\
\hline Male & 5 & Philic & 1 & UNI & BERG syn \\
\hline Male & 6 & Philic & 2 & BIL & \\
\hline Female & 6 & Philic & 1 & UNIL & truamatic cataract \\
\hline Female & 8 & Philic & 2 & UNI & done outside \\
\hline Male & 8 & Philic & 2 & UNI & TRUAMATIC CATARACT \\
\hline Male & 8 & Philic & 1 & BIL & \\
\hline Female & $11 y$ & Philic & 1 & BIL & \\
\hline Female & 4 & Phillic +30 & 3 & UNI & IOL-EXPLANT \\
\hline Male & & Phobic & 1 & BIL & $\begin{array}{l}\text { DIED } 21 \backslash 2 \mathrm{Y} \\
\mathrm{REF}+4\end{array}$ \\
\hline Female & 4 & Phobic & $2 \mathrm{X}$ & BIL & $\begin{array}{l}\text { left IOL dropped in vitrous } \\
\text { right membranectomy } \\
1 \backslash 12\end{array}$ \\
\hline Female & 2 & Phobic & 1 & BIL & \\
\hline Female & 4 & Phobic & 1 & BIL & \\
\hline Male & 2 & Phobic & 1 & BIL & \\
\hline Male & 5 & Phobic & 1 & UNIL & \\
\hline
\end{tabular}




\begin{tabular}{|c|c|c|c|c|c|}
\hline Male & 4 & Phobic & 2 & BIL & \\
\hline Male & 4 & Phobic & 1 & BIL & \\
\hline Female & 6 & Phobic & 1 & UNI & va $0.5+$ \\
\hline Male & 4 & Phobic & 1 & UNI & \\
\hline Male & 9 & Phobic & 1 & BIL & $\begin{array}{l}\text { VA } .9 \\
\text { LAST REF }+2.5\end{array}$ \\
\hline Male & 6 & Phobic & 1 & BIL & \\
\hline Male & 9 & Phobic & 2 & UNI & $\begin{array}{l}\text { LEFT TRUAMATIC CATRACT } \\
\text { NOT USING DROPS } \\
\text { DEVELOPED PUPILLARY MEMBRANE 2W SX } \\
\text { REF + } 1 \text { VA } 0.4\end{array}$ \\
\hline Male & 9 & Phobic & 1 & BIL & \\
\hline Female & 12 & Phobic & 1 & BIL & final va 0.9 \\
\hline Male & 10 & Phobic & 1 & BIL & \\
\hline Female & $13 y$ & Phobic & 1 & BIL & VA 1.0 \\
\hline Male & 14 & Phobic & 1 & BIL & $\begin{array}{l}\text { bil cat } \\
\text { steriod for v. catter? } \\
\text { Bil glucoma } \\
\text { bil phako } \\
\text { bil ahemd valve }\end{array}$ \\
\hline Male & 18 & Phobic & 1 & BIL & down syndrome \\
\hline Female & 18 & Phobic & 1 & BIL & $\begin{array}{l}\text { YAG PCO } \\
2 \text { Y AFTER SX } \\
\text { didmoad }\end{array}$ \\
\hline Female & & PMMA & & UNIL & $\begin{array}{l}\text { TRUAMATIC CATARACT } \\
\text { APHAKIC 2ND IMP } 6 \text { M LATER }\end{array}$ \\
\hline Male & $13 \mathrm{Y}$ & & 1 & BIL & $\begin{array}{l}\text { PCO } 7 \backslash 11\{6 \text { M AFTER SX } \\
\text { BIL-YAG LASER }\end{array}$ \\
\hline Male & 11 & & 1 & UNI & TRUAMATIC CATARACT \\
\hline
\end{tabular}

\section{Copyrights}

Copyright for this article is retained by the author(s), with first publication rights granted to the journal.

This is an open-access article distributed under the terms and conditions of the Creative Commons Attribution license (http://creativecommons.org/licenses/by/4.0/). 